\title{
NANO ACCURACY ELEVATION OF ULTRA-PRECISION MACHINING USING OPTICAL FIBER LASER ENCODER SYSTEM*
}

\author{
Geon Lee* \\ Dept. of Advanced Parts \& Materials Engineering, Graduate School of Chosun University, 375, Seosuk-Dong, \\ Gwangju 501-759, South Korea \\ imlk31@naver.com \\ Sung-Hyun $\mathrm{Kim}^{\dagger}$ \\ Dept. of Advanced Parts \& Materials Engineering, Graduate School of Chosun University, 375, Seosuk-Dong, \\ Gwangju 501-759, South Korea \\ ddr0309@hanmail.net \\ Nam-Su Kwak \\ Dept. of Advanced Parts \& Materials Engineering, Graduate School of Chosun University, 375, Seosuk-Dong, \\ Gwangju 501-759, South Korea \\ kns8679@gmail.com \\ Jae-Yeol Kim ${ }^{\S}$ \\ Dept. of Mechatronics Engineering, Chosun University, 375, Seosuk-Dong, Gwangju 501-759, South Korea \\ jykim@chosun.ac.kr
}

\begin{abstract}
The ultra-precision products which recently experienced high in demands had included the large areas of most updated technologies, for example, the semiconductor, the computer, the aerospace, the media information, the precision machining. For early $21^{\text {st }}$ century, it was expected that the ultra-precision technologies would be distributed more throughout the market and required securing more nation-wise advancements. Furthermore, there seemed to be increasing in demand of the single crystal diamond tool which was capable of the ultra-precision machining for parts requiring a high degree of complicated details which were more than just simple wrapping and policing. Moreover, the highest degree of precision is currently at $50 \mathrm{~nm}$ for some precision parts but not in all. The machining system and technology should be at very high preformed level in order to accomplish this degree of the ultra-precision.
\end{abstract}

Keywords: Ultra-Precision Cutting Unit(UPCU); PZT; Optical fiber laser encoder; dual positioning control ; Matlab Simulink.

\section{Introduction}

In this $21^{\text {st }}$ century, the world is preparing new revolution of knowledge-based society after the industrial society, and the world concentrates on information Technology, Nano

\footnotetext{
${ }^{*}$ Corresponding author/ TEL: +82-62-230-7745, FAX: +82-62-230-7035.

${ }^{\dagger}$ Corresponding author/ TEL: +82-62-230-7745, FAX: +82-62-230-7035.

${ }^{\ddagger}$ Corresponding author/ TEL: +82-62-230-7745, FAX: +82-62-230-7035.
} 
each of which use is different, and which is accepted in each field, such as, science, industry, media, etc. Therefore, this paper proposes a method of producing an ultraprecision positioning machine by combining a micro stage and a global stage to meet the request in the field. Also, this paper also propose a method of designing an optimum controller, using a minimum order observer with dual servo control scheme to which a modern control technique is applied.

\section{System configuration}

The system consists of an optical fiber laser encoder unit and an optical fiber laser detector head with Fig. 1 and Laser encoder unit sends two Flexible optical fibers by detector head to laser beam. Optical fiber laser detector head generating feedback signal that do laser beam that is reflected in plane mirror to do return by unit by encoder again and need in position control. Inside of optical fiber laser encoder unit is consisted of electronic circuit that laser light source (He-Ne) and 2 congratulations position control are available. This research was intended to confirm effectiveness of environment error compensation measuring zero point measurement of laser encoder and frequency of laser encoder by environment change.
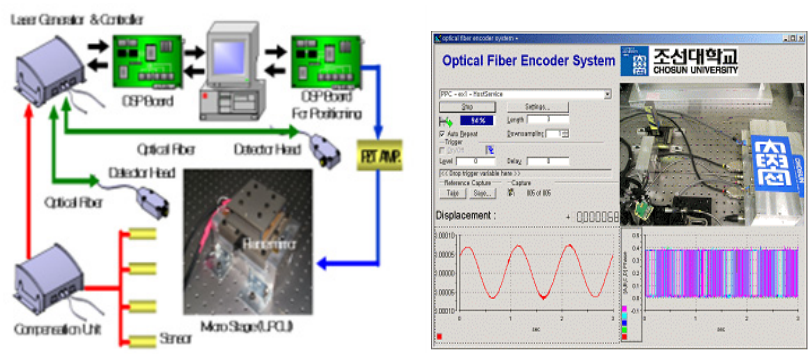

Fig. 1. Optic fiber laser encoder system and UPCU

\section{Optical fiber laser encoder system}

\subsection{The environmental compensation of the optic fiber laser encoder}

This investigation had compensated the air reflective index at the real-time by measuring the air pressure and the temperature when the laser beam had passed thought the air and applying the environment compensation unit which could compensate for the thermal expansion of the material and the device structure. The environmental unit was designed to output the digital signal and analog signals of various resolutions by receiving input of the differentiated digital quadrature signal of RS422 format on the basis of the high performance DSP technology. In this investigation, the digital signal was selected. The wavelength of laser varies very slightly depending on the refractive index of the air it travels thought. The refractive index of the depends primarily on it's temperature, pressure and humidity. Any of the following changes in the air will increase the wavelength by $0.25 \mathrm{ppm}$ (part per million). 


\section{Constructing servo system}

\subsection{Constructing 2 input 1 output control system}

In this paper, assuming the ultra-precision $\mathrm{X}-\mathrm{Y}$ stage is a 2 input 1 output system, an optimum control system was examined using the modern control theory. Since the system design by the classical control theory is based on trials and errors, it is thus not an optimum control system. On the contrary, with the system design using modern control theory by the state-space scheme, designers can design a system having a closed loop polarity (desired characteristic equation) or an optimum control for a given performance index. The modern control theory allows a designer to include initial conditions in design if required. However, design by the modern control theory thought the state-space scheme requires mathematical representation for dynamic characteristics of a system. This is different from the classical scheme that experimental frequency response curves without sufficient accuracy can be used in design without mathematical representations. Accordingly, in this paper, with the advantages of such modern control theory, the 2 input 1 output state equation of ultra-precision plane $\mathrm{X}-\mathrm{Y}$ was derived on the basis of global servo and micro stage modeling. The state equation of the ultra-precision plane $\mathrm{X}$ $\mathrm{Y}$ equation is described below. By doing so, it is possible to represent tools with one state equation and to design a global servo and a UPCU in an integral body. On the basis of the state equation (1), the 1-type optimum servo system is designed. Among six state variables used in the designed 1-type optimum control system can be measured of the minimum order observer. On the basis of such a theoretical basis, simulation was repeated to design a control system in which micro-servo's length was not saturated and amp power current was not saturated.

$$
\begin{aligned}
& {\left[\begin{array}{cccccc}
0 & 1 & 0 & 0 & 0 & 0 \\
a_{1} & a_{2} & a_{3} & a_{24} & a_{2} & 0
\end{array}\right] \quad\left[\begin{array}{cc}
0 & 0 \\
b_{21} & 0
\end{array}\right] \quad x_{s}=\left[\begin{array}{llllll}
x & \dot{x} & \boldsymbol{v} & \boldsymbol{x}_{c} & \dot{x}_{c} & i_{m}
\end{array}\right]^{T}} \\
& \dot{\boldsymbol{x}}_{s}=\left[\begin{array}{cccccc}
a_{21} & a_{22} & a_{23} & a_{24} & a_{25} & 0 \\
0 & 0 & a_{33} & 0 & 0 & 0 \\
0 & 0 & 0 & 0 & 1 & 0 \\
0 & 0 & 0 & 0 & a_{55} & a_{56} \\
0 & 0 & 0 & 0 & a_{65} & a_{66}
\end{array}\right] \boldsymbol{x}_{s}+\left[\begin{array}{cc}
b_{21} & 0 \\
b_{31} & 0 \\
0 & 0 \\
0 & 0 \\
0 & b_{62}
\end{array}\right]\left[\begin{array}{l}
V_{p} \\
V_{m}
\end{array}\right] \begin{array}{l}
y=\left[\begin{array}{ccccc}
1 & 0 & 0 & 0 & 0 \\
0 & 0 & 0 & 1 & 0
\end{array}\right] x_{s} \\
y=\left[\begin{array}{lll}
1 & 0
\end{array}\right] \dot{y}
\end{array} \\
& a_{21}=-\frac{k_{p}}{m}, a_{22}=-\frac{C_{p}}{m}, a_{23}=\frac{k_{p} k_{p}}{m}-\frac{C_{p} k_{p}}{R C_{m}} a_{24}=-\frac{\boldsymbol{k}_{p}}{m}, a_{25}=\frac{C_{p}}{\boldsymbol{m}}, a_{33}=-\frac{1}{R_{\varepsilon}} \\
& a_{55}=-\frac{C_{s}}{J+k^{2} M}, a_{56}=-\frac{k k_{2}}{J+k^{2} M}, a_{55}=-\frac{\boldsymbol{k}_{1}}{L_{m} k} \quad a_{66}=-\frac{\boldsymbol{R}_{m}}{L_{m}}, \quad b_{21}=\frac{C_{p} K_{p}}{R C_{m}} G_{m}, \quad b_{31}=\frac{K_{m}}{R_{c}}, \quad b_{62}=\frac{G_{p}}{L_{m}}
\end{aligned}
$$

\subsection{Designing minimum order observer}

Some state variables in an actual system can be directly measured, so that it is not required to estimate them. Assuming $\mathrm{n}$ is a state variable order, the observer for estimating state variables smaller than $\mathrm{n}$ is called a minimum order observer. Say the state vector, and power $\mathrm{y}$ is $\mathrm{m}$ vector. Since $\mathrm{m}$ output varibles are represented by means of a linear combination of state variables. Therefore, the n-m order observer is the minimum order observer. The order of state variables in this system is 6 th order, and the measurable output vector is first order. As a result, the state variable $\mathrm{x}$ can be measured 
by a laser interferometer, and the remaining state variable $\dot{\mathrm{x}}, v, \dot{\mathrm{x}}_{\mathrm{c}}, \mathrm{i}_{\mathrm{m}}$ are estimated from the minimum order observer. Fig. 2 shows a minimum order applied to this system.

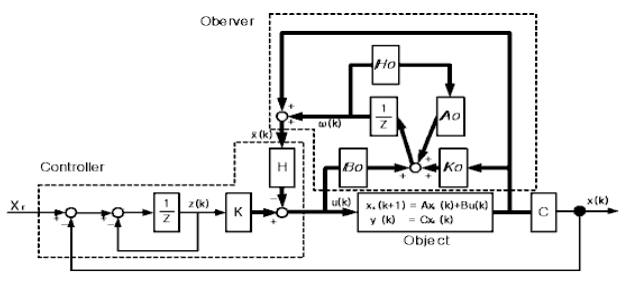

Fig. 2. Block diagram of UPCUusing minimum order observer

\section{Simulation and real-time control}

In testing ultra-precision positioning of the system, simulation and real-time control was carried out with MATLAB SIMULINK. The experiment was a single servo experiment and a dual servo control test. In the single servo control test, simulation and real-time control was carried out with a PID controller for both of a global servo and a micro servo, respectively. For the dual servo control test, the minimum order observer using the modern control theory was applied to compare the results of simulation and real-time control.

\subsection{Simulation}

For dual servo simulation, the minimum order observer of the modern control theory was applied to the state equation of 2 input 1 output derived in the above to construct a 1 type optimal servo system and the system was applied to MATLAB SIMULINK. Fig. 4 (a) shows a dual servo SIMULINK and (b) shows SIMULINK of a minimum order observer. Fig. 3 (a) shows the displacements of a dual state including a global servo and a micro servo, and the displacement of the global stage when carrying out dual servo simulation by means of SIMULINK. Fig. 3 (b) shows the displacement of a micro servo. Here, it is seen that the system is driven while the error of the global stage is corrected by the micro servo. This means that correct gang control is operating. Tables 1 show control performance by simulation of a global servo and a micro servo.

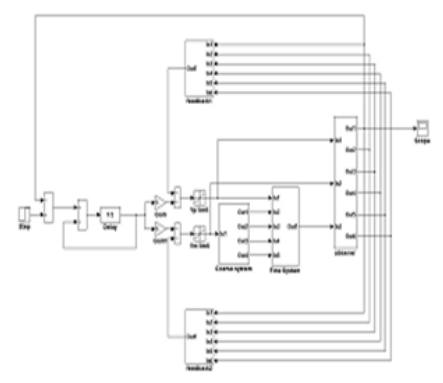

(a) The displacements of a dual state including a global servo and a micro servo

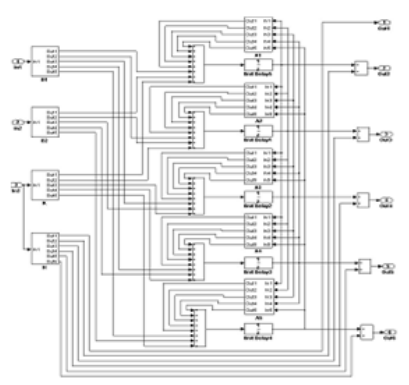

(b) The displacement of a micro servo

Fig. 3 SIMULINK of dual servo for Ultra-precision single plane X-Y and Minimum order observer subsimulink of dual servo for Ultra-precision single lane X-Y stage 


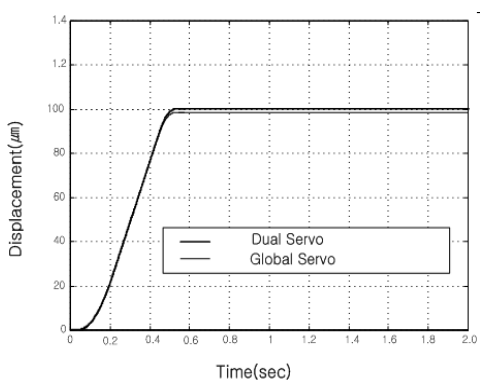

(a) Dual servo(UP2)

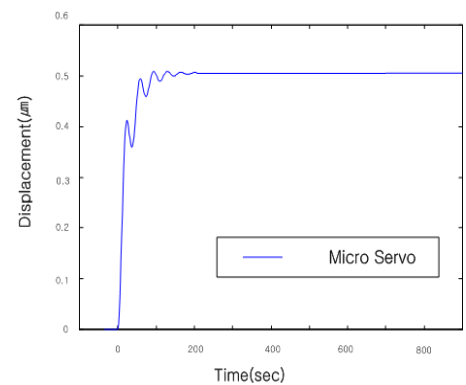

(b) UPCU

Fig. 4. Step response of perform simulation

Table 1. Step response of global servo for simulation and micro servo

\begin{tabular}{llll}
\hline Definition & Value & Definition & Value \\
\hline Steady-state error & 0 & Steady-state error & 0 \\
Maximum overshot[\%] & $0.1(10 \%)$ & Maximum overshot[\%] & $0.1(10 \%)$ \\
Rise Time[s] & 0.047 & Rise Time[s] & 0.005 \\
Setting Time[s] & 0.062 & Setting Time[s] & 0.0075 \\
\hline
\end{tabular}

\subsection{Real-time control}

A PID controller was applied to a global servo and a micro servo to carry out positioning test. As a result of the test, satisfactory performance was found in each tool. Through the experiment and result, we could see stability of the global servo and the micro servo. By applying the minimum order observer based of the modern control theory for ultraprecision position control, real-time control of a dual servo was carried out. Fig. 6 show resolution experiment of a dual servo. It is seen that the resolution of each axis is about $\pm 10 \mathrm{~nm}$. Fig. 7 shows $2 \mathrm{D}$ trace of two axes $\mathrm{X}-\mathrm{Y}$. The resolution of the tool obtained through the above experiment was less than $\pm 10 \mathrm{~nm}$.

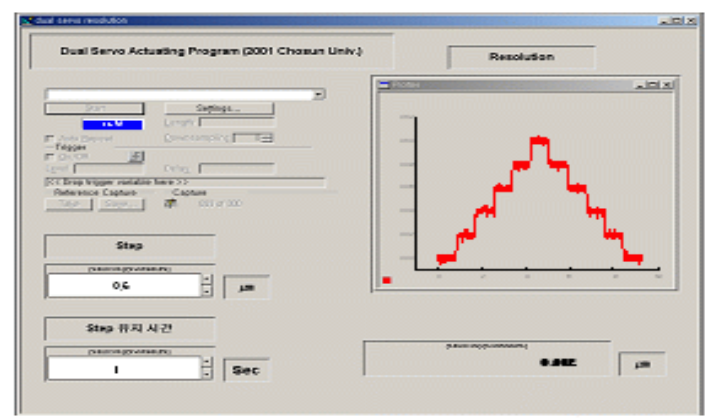

Fig. 5. X-axis RTI sub-simulink of dual servo and Resolution layout of ControlDesk for dual servo 


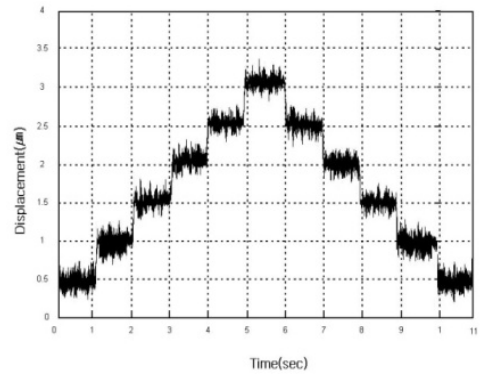

Fig. 6. Resolution experiment of X-axis and Y-axis dual positioning control

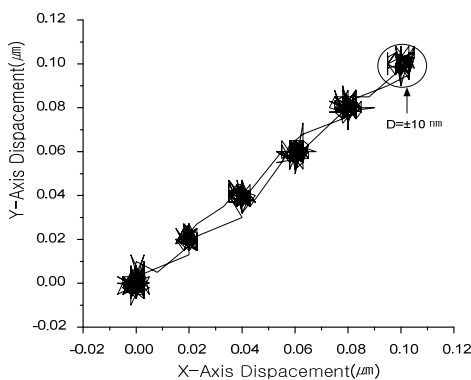

Fig. 7. Resolution experiment of $\mathrm{X}-\mathrm{Y}$ axis dual positioning control

\section{Conclusion}

The results of research for designing an optimum controller of a ultra-precision plane XY stage, performed in this paper, is described below

1. Performance evaluation of a controller was carried out through simulation using Matlab for the control system of a servo tool using in a plane X-Y stage ultra-precision positioning system. We devised a dual servo control algorithm using a minimum order observer to which the modern control theory is applied through comparing and analyzing the conventional control algorithm. We could see stability and good performance of the controller.

2. We compared and analyzed the simulation result and the ultra-precision positioning experiment result through a real-time ultra-precision positioning experiment using Matlab Simulink and Control Desk of dSPACE. We could see stability and good performance of the position control algorithm and the plane $\mathrm{X}-\mathrm{Y}$ stage ultra-precision positioning system.

\section{References}

1. S,T,Smith. And D,G,Chetwynd., 1992, Foundation of Ultra precision mechanism design(Gordon and Breach Science Publishers)

2. Thomas J. R. Hugher, "The Finite Element Method," Prentice-Hall International

3. H, Nakazawa, 1994, principles of precision engineering Editions, pp.90-97,, 1987.

4. Shiraishi M., Uehara K., "In-Process Control of Workpiece Dimension in Turning," Annal of The CIRP, Vol. 28, pp.333-337., 1979.

5. Lee E, S., Lee J. K., Suto T., :A Simulation System for the Eatablishment of Grinding Operation Standards," JSPE, Vol. 54 No. 12, pp. 81-86., 1988 